\title{
Atypical and Rare Forms of Cutaneous Lupus Erythematosus: The Importance of the Diagnosis for the Best Management of Patients
}

\author{
Astrid Herzum ${ }^{a}$ b Giulia Gasparini ${ }^{a, b}$, c Emanuele Cozzani ${ }^{a}$, b \\ Martina Burlando ${ }^{a, b}$ Aurora Parodi ${ }^{a, b}$ \\ aSection of Dermatology, Department of Health Sciences, University of Genoa, Genoa, Italy; bermatology Unit, \\ Ospedale Policlinico San Martino IRCCS, Genoa, Italy; 'Department of Specialistic Medicine, University of Genoa, \\ Genoa, Italy
}

\section{Keywords}

Cutaneous lupus erythematosus · Bullous lupus

erythematosus · Hypertrophic discoid lupus

erythematosus · Lupus mastitis · Lupus comedonicus

\begin{abstract}
Lupus erythematosus (LE) is an autoimmune disease with a wide range of clinical and cutaneous manifestations. Along with the well-known typical cutaneous manifestations of $L E$, some cutaneous manifestations are rarer, but still characteristic, enabling the dermatologist and the general practitioner who know them to suspect cutaneous LE (CLE) and investigate a possible underlying systemic involvement. Indeed, not infrequently a skin manifestation is the first presentation of systemic LE (SLE), and $>75 \%$ of SLE patients show signs of skin disease during the course of the illness. Especially, SLE involvement occurs in cases of acute CLE, while it is uncommon in subacute CLE and rare in chronic CLE. This review aims to concentrate especially on atypical cutaneous manifestations of $L E$ to enable the clinician to diagnose even the rarest forms of CLE. ๑2021 S. Karger AG, Basel
\end{abstract}

karger@karger.com

(c) 2021 S. Karger AG, Basel

www.karger.com/drm

Karger"

\section{Introduction}

Lupus erythematosus (LE) is an autoimmune disease with an extraordinarily wide range of clinical and cutaneous manifestations. The clinical spectrum of cutaneous lupus erythematous (CLE) ranges from isolated plaques to widespread lesions, and the symptoms and clinical course of CLE vary widely among patients.

Along with the well-known typical cutaneous manifestations of LE, some are rarer but still characteristic, enabling the clinician who knows them to suspect CLE. This is why general practitioners and dermatologists should be aware of the rare and atypical forms of CLE, in order to diagnose LE and investigate a possible underlying systemic involvement.

Indeed, CLE can present as an isolated skin disease or as a manifestation of systemic LE (SLE). The incidence of isolated CLE in the USA and in Europe is 4 cases per 100,000 inhabitants per year, and SLE occurs in 3 cases

\section{A. Herzum and G. Gasparini contributed equally to this paper.}

Correspondence to:

Astrid Herzum, astridherzum@yahoo.it 


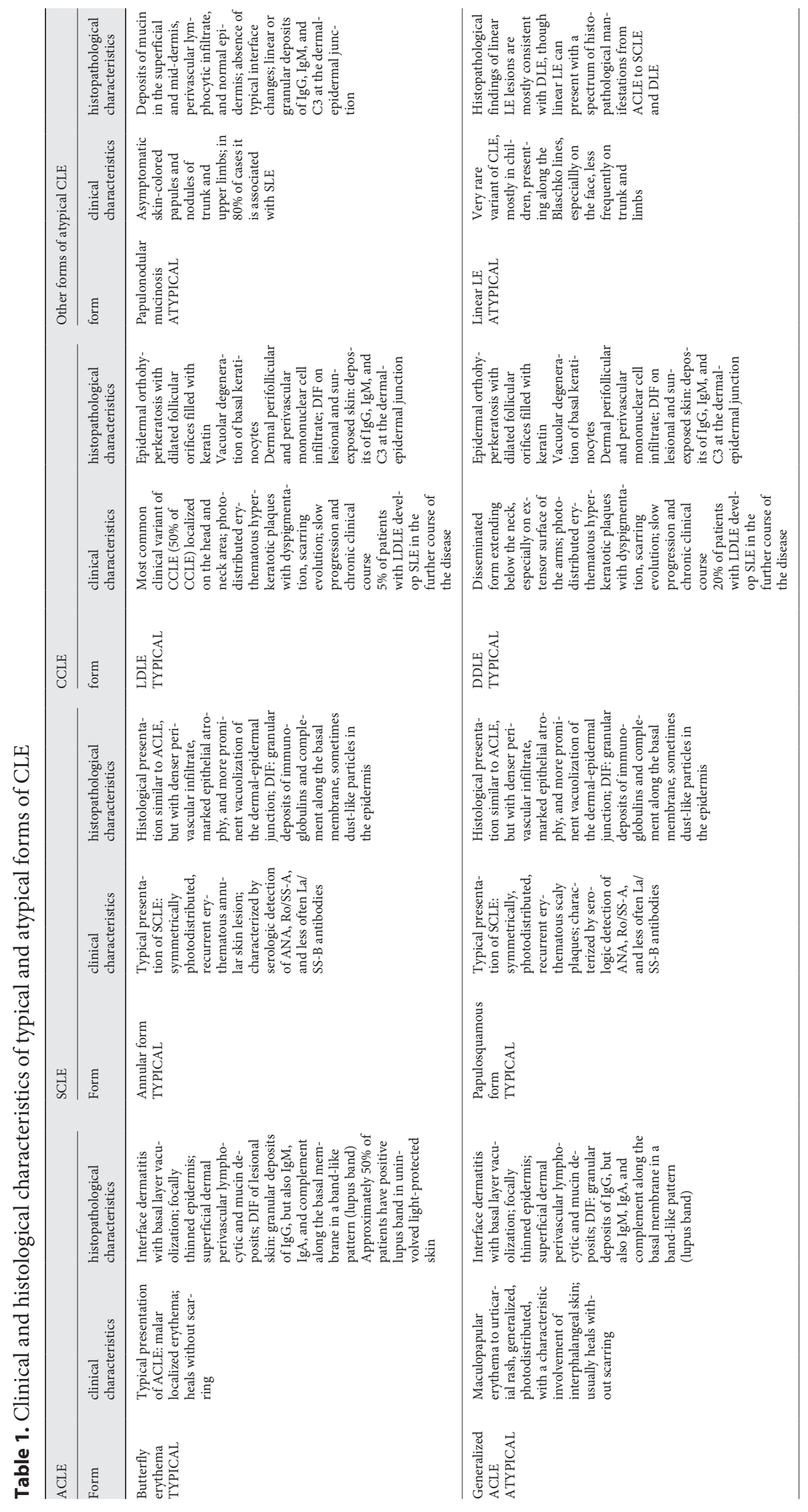




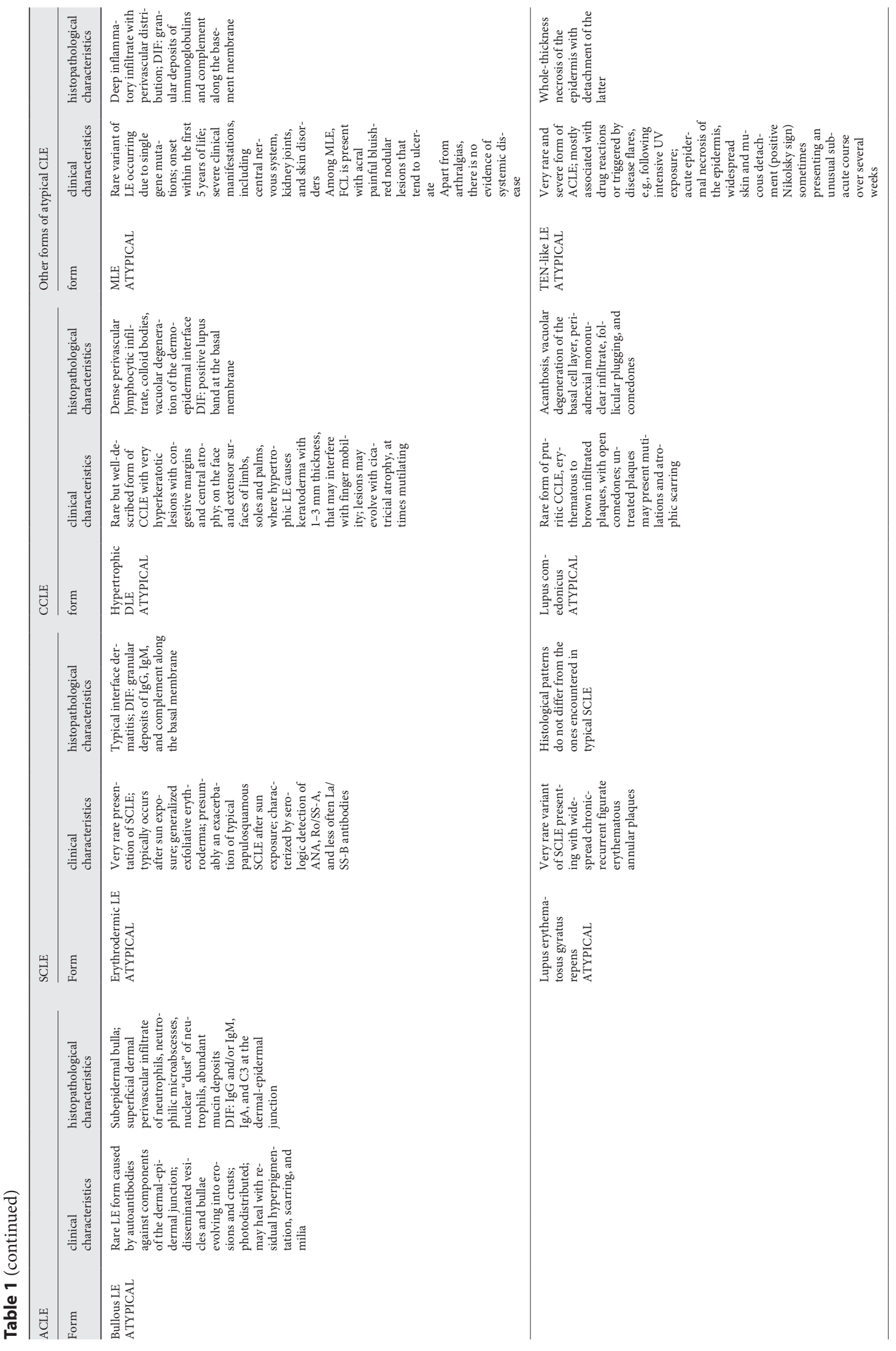




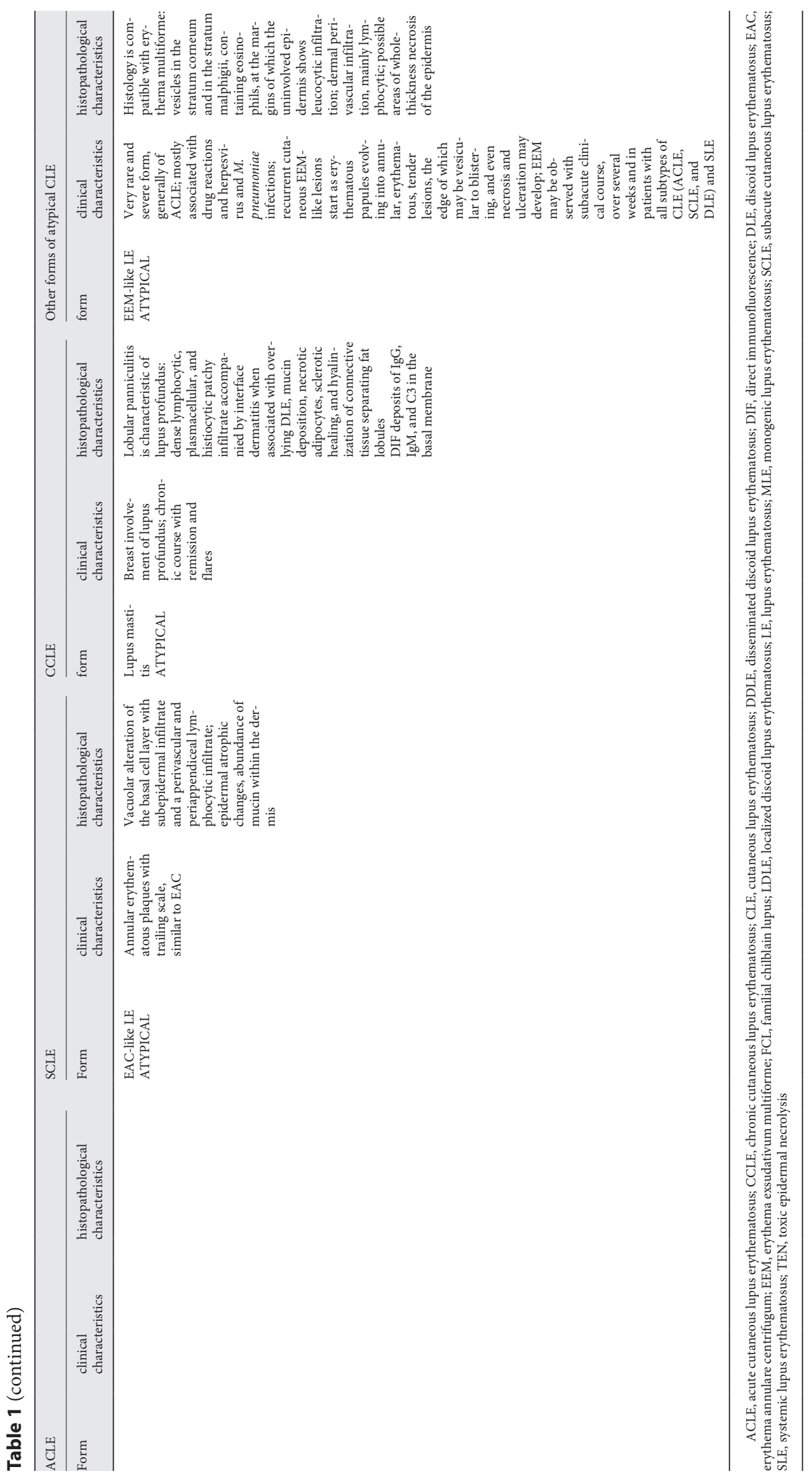




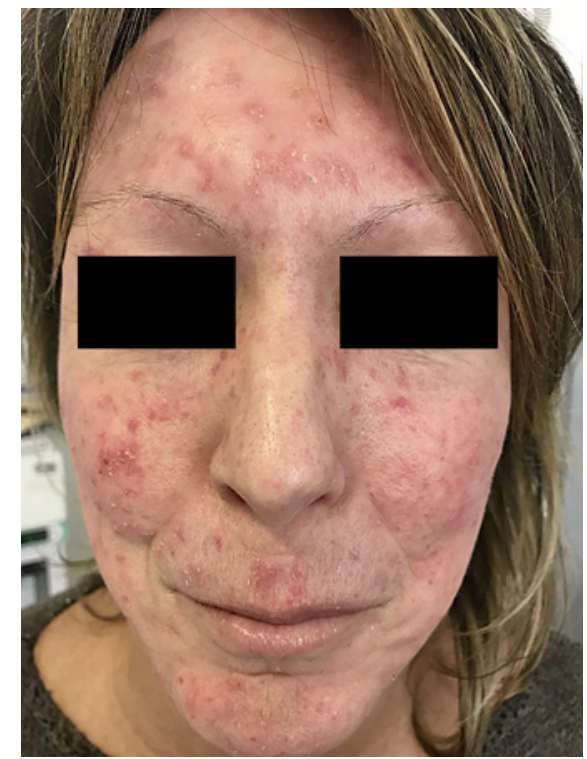

Fig. 1. Generalized acute cutaneous lupus erythematosus: erythematous macules and papules on sun-exposed skin of the face.

per 100,000 inhabitants per year $[1,2]$. In SLE patients, skin manifestations are present in $70-80 \%$ of cases and are a first sign of SLE in almost $25 \%$ of cases $[1,2]$. Indeed, in regards to the different clinical presentation, CLE can be subdivided into (1) acute CLE (ACLE), an acute form with rapid development of cutaneous manifestations and acute clinical course, often associated with systemic involvement, (2) subacute CLE (SCLE), a subacute form with subacute clinical course, over several weeks, and (3) chronic CLE (CCLE), a chronic form characterized by slow progression and chronic clinical course of the duration of months to years that rarely involves internal organs $[1,2]$. Systemic involvement occurs in almost $90 \%$ of ACLE cases, in $20-30 \%$ of SCLE cases, and in $<5 \%$ of localized chronic discoid LE (CDLE) cases $[1,2]$.

To date, the classification of CLE proposed by Gilliam and Sontheimer in 1981 is still used to divide CLE into LE-specific and LE-nonspecific skin conditions [2,3], depending on the presence or absence of an interface dermatitis on histopathological examination, which is represented by a cytotoxic, anti-epithelial inflammation at the dermal-epidermal junction, characterized by hydropic degeneration, colloid bodies, and death of keratinocytes [3]. Even though this classification falls short for some CLE variants, such as lupus erythematosus panniculitis, which presents no interface dermatitis, these variants are still considered proper CLE-specific lesions.

Atypical Cutaneous Lupus Erythematosus

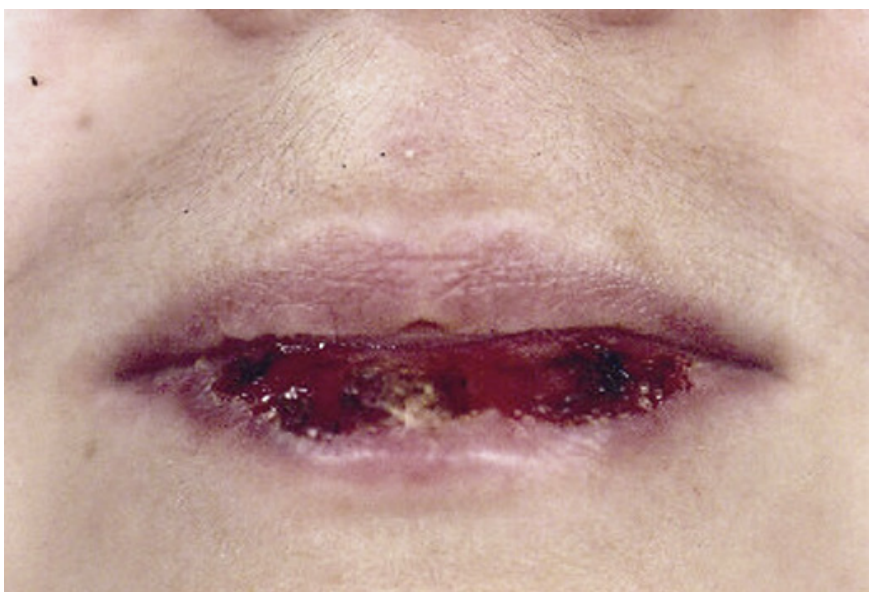

Fig. 2. Mucous involvement during generalized acute cutaneous lupus erythematosus with ulceration of the labial mucosa.

Hereby we will discuss the clinical and histopathological presentation of rare and atypical variants of LE-specific skin conditions and review possible differential diagnoses.

\section{Rare Forms of ACLE}

ACLE is usually associated with SLE and presents often as its first manifestation. It typically occurs as a classic localized butterfly erythema, but other rarer manifestations include generalized photodistributed LE and bullous LE (Table 1) $[4,5]$.

\section{Generalized ACLE}

The generalized manifestation of ACLE presents as a maculopapular or urticarial rash, especially on sun-exposed skin (Fig. 1), with characteristic involvement of the extensor aspects of the fingers and sparing of the knuckles. This is important to make a differential diagnosis from the Gottron's papules of dermatomyositis that spare the interphalangeal skin, presenting with similar purple papules to plaques but localized over the top of the hands' small joints. Also, drug-induced and viral rashes represent potential differential diagnoses that must be considered. ACLE lesions usually heal without scarring, but postinflammatory hyper- or hypopigmentation is possible [2]. Involvement of mucous membranes, especially with ulcerative progression, is not infrequent (Fig. 2) [2]. On histopathological investigation, ACLE presents characteristic interface dermatitis with basal layer vacuolization and sometimes focally thinned epidermis and superficial der- 


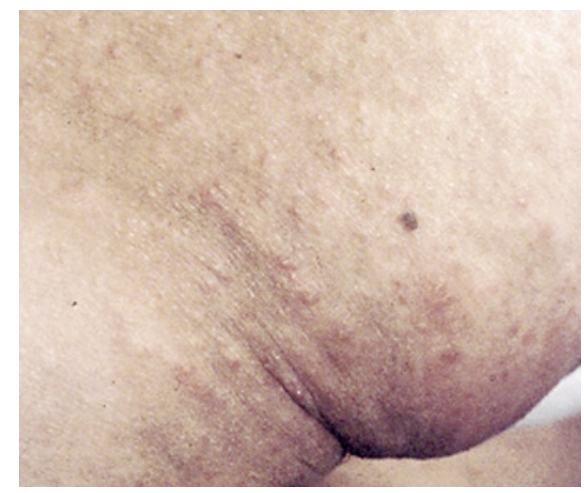

Fig. 3. Bullous lupus erythematosus: disseminated vesicles and crusts on erythematous to clinically normal skin, accompanied by residual hyperpigmentation and milia.

mal perivascular lymphocytic and mucin deposits in the reticular dermis $[4,5]$. Direct immunofluorescence (DIF) of lesional skin shows a characteristic deposition of immunoglobulins along the dermal-epidermal junction defined as lupus band test. This consists of granular deposits of IgG, but also IgM, IgA, and complement along the basal membrane in a band-like pattern (lesional lupus band) [5]. Also, approximately $50 \%$ of patients have positive lupus band in uninvolved light-protected skin.

\section{Bullous LE}

Bullous LE is a variety of LE caused by autoantibodies against collagen VII or other components of the dermalepidermal junction $[5,6]$. It is a rare entity, usually observed in ACLE with increased systemic disease activity. It is a subepidermal blistering process with disseminated vesicles and bullae arising prevalently on sun-exposed skin, on erythematous or clinically normal skin; also erosions and crusts can be observed and mucosal membranes can also be affected. Sometimes lesions heal with residual hyperpigmentation, scarring, and milia (Fig. 3) $[4,5]$. Notably, this entity must be differentiated from blisters which can occur in ACLE or SCLE secondary to vacuolar degeneration of the basal layer of the epidermis.

Histopathological findings in bullous LE show a subepidermal bulla with superficial perivascular infiltrate containing neutrophils accompanied by neutrophilic microabscesses in the papillary dermis. Interestingly, nuclear "dust" of neutrophils in the upper dermis, absence of eosinophils, and abundant mucin deposits are clues for the differential diagnosis of bullous LE from Duhring dermatitis herpetiformis, linear IgA dermatosis, and epidermolysis bullosa acquisita [5]. DIF shows IgG and/or $\operatorname{IgM}$, IgA, and C3 at the dermal-epidermal junction [4].

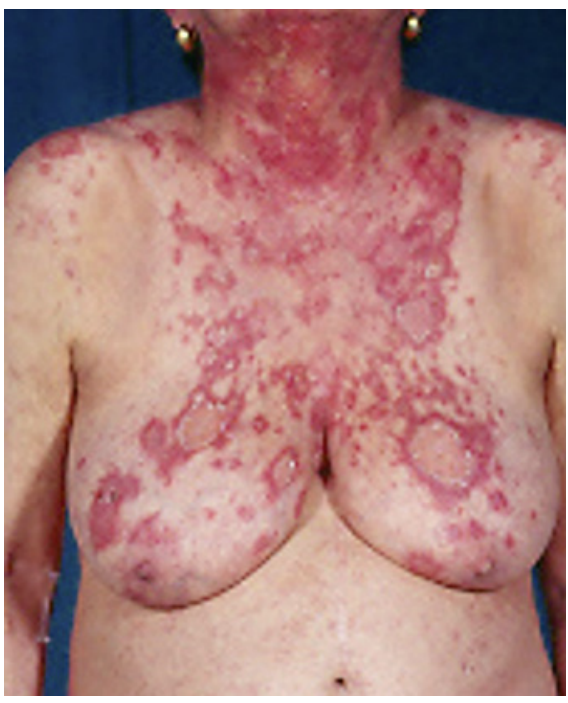

Fig. 4. Annular subacute cutaneous lupus erythematosus: symmetrical erythematous annular skin lesions distributed on the sunexposed skin of the chest and neck.

Sera from patients with bullous LE often contain autoantibodies towards type VII collagen, commonly known as the epidermolysis bullosa acquisita antigen, and some patients also have other autoantibodies in their sera, as for example autoantibodies directed against the epidermal side of salt split skin [4], permitting to classify different subsets of bullous LE depending on autoantibodies reacting with the amino-terminal noncollagenous domain type 1 and 2 (NC1 and NC2) of collagen VII, against laminin 332, laminin 311, and bullous pemphigoid antigen 1 (BP230) [5].

Interestingly, rare variants of CLE may also coexist in the same patient with findings that combine more than one atypical cutaneous variant of LE, as presented by $\mathrm{Da}$ Silva Sousa et al. [7] who presented an utmost rare case of bullous LE with an erythema gyratum repens-like pattern, as we will describe further.

\section{Rare Forms of SCLE}

Typical SCLE forms are the annular (Fig. 4) and papulosquamous ("psoriasiform") (Fig. 5) forms which are characterized by photosensitivity and symmetrical distribution on sun-exposed areas, with recurrent skin lesions $[3,4]$. The histological presentation is similar to that of ACLE. The perivascular infiltrate is denser, there is marked atrophy of the epithelium and more prominent vacuolization of the dermal-epidermal junction. The bas- 


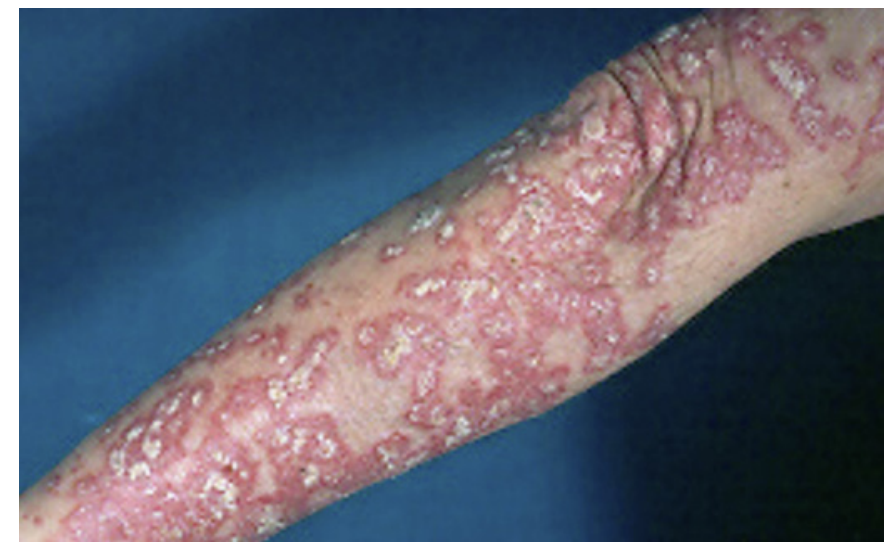

Fig. 5. Psoriasiform subacute cutaneous lupus erythematosus: erythematous scaly plaques photodistributed on the upper limb.

al membrane may be thickened. At DIF, granular deposits of immunoglobulins and complement along the basal membrane are typical, and sometimes dust-like particles are present in the epidermis. Rare variants of the SCLE include erythrodermic LE, lupus erythematosus gyratus repens, and erythema annulare centrifugum-like LE (Table 1) [5].

\section{Erythrodermic LE}

Erythrodermic LE has been reported as a very rare presentation of SCLE. It manifests with generalized exfoliative erythroderma, typically after sun exposure. Presumably, it may represent an exacerbation of typical papulosquamous SCLE after sun exposure. Histological findings present characteristic interface dermatitis. At DIF, granular deposits of IgG, IgM, and complement along the basal membrane are typical. Serologic evaluation with detection of ANA, Ro/SS-A, and La/SS-B antibodies usually confirms the diagnosis [8].

\section{Lupus Erythematosus Gyratus Repens}

Lupus erythematosus gyratus repens is an annular variant of SCLE, presenting with widespread chronic-recurrent figurate erythematous plaques. Only a few cases have been described in the literature. The clinical and histological patterns do not differ from the ones encountered in SCLE [9].

\section{Erythema Annulare Centrifugum-Like LE}

SCLE may present with annular lesions that mimic erythema annulare centrifugum with its annular erythematous plaques with trailing scale. Histology findings include the typical vacuolar alteration of the basal cell layer

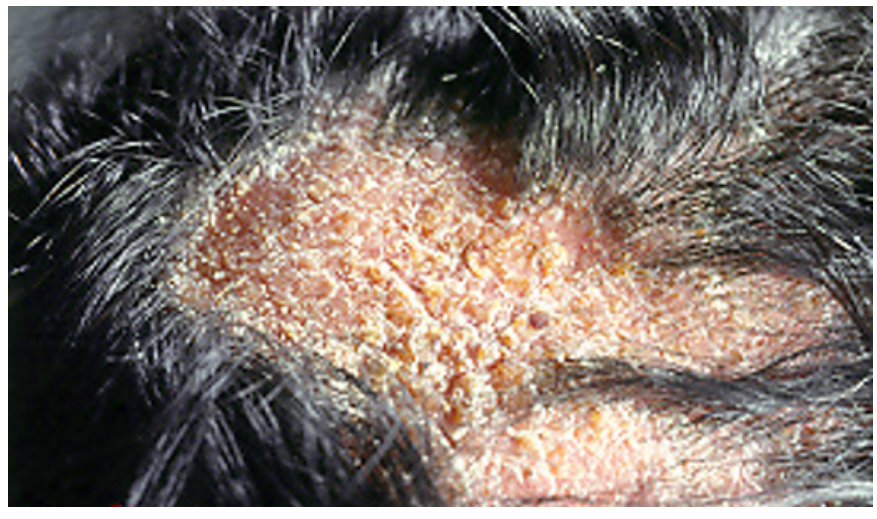

Fig. 6. Hypertrophic lupus erythematosus: adherent keratotic yellowish plaque on the scalp.

with subepidermal infiltrate and a perivascular and periappendiceal lymphocytic infiltrate. Epidermal atrophic changes are common, as is abundance of mucin within the dermis [10].

\section{Rare Forms of CCLE}

CCLE is characterized by a slow progression and by a chronic clinical course (months to years) [1]. Discoid LE (DLE) represents the most common clinical variant of CCLE (50\% of CCLE) and occurs mainly in the head and neck area, representing localized DLE (LDLE) $[1,5,11$, 12].

The disseminated form of DLE extends below the neck, especially on the extensor surface of the arms. Overall, only approximately $50 \%$ of CDLE patients are ANApositive, but in fact only $5-20 \%$ develop systemic features of SLE [1]. A distinction between LDLE and disseminated DLE (DDLE) is prognostically important: $20 \%$ of patients with DDLE, but only $5 \%$ of patients with LDLE develop SLE in the further course of the disease $[2,13]$.

Less common variants of CCLE are hypertrophic DLE, lupus mastitis, lupus comedonicus, and for some authors also lupus erythematosus tumidus (Table 1) [1, 11].

\section{Hypertrophic DLE}

A rare but well-described variety of DLE is hypertrophic or verrucous DLE, in which very pronounced hyperkeratosis is characteristic [2]. Hypertrophic DLE was at first named "cretaceous herpes" by Devergie [14] for the congestive margins and central atrophy that accompany the hypertrophic lesions. Only in 1950 did Bechet [15] identify and describe it as a subtype of CLE. 


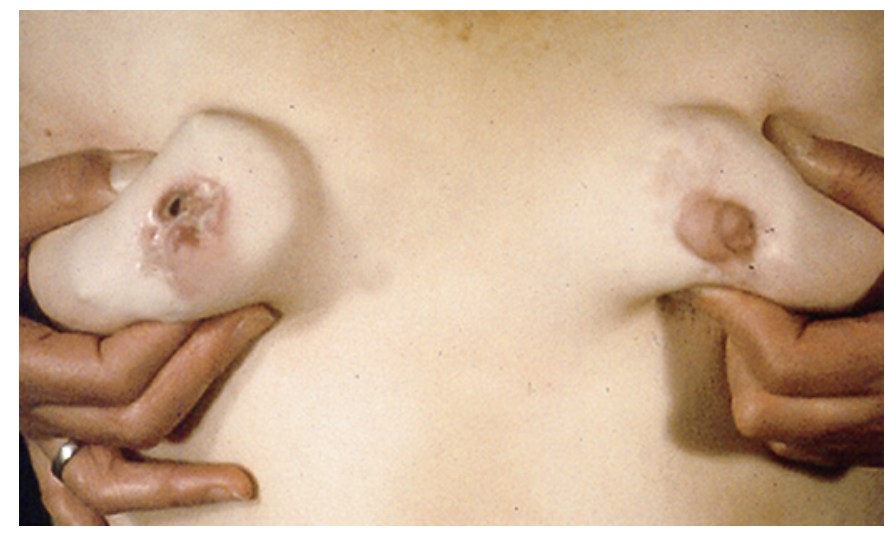

Fig. 7. Lupus mastitis: erythematosus painful deep nodules of the breast.

Hypertrophic DLE is rare, representing only $2 \%$ of CCLE, but it may be associated with every form of CLE [16]. The lesions typically occur on the face and on the extensor surfaces of limbs, soles, and palms, appearing as single or multiple papular or nodular lesions covered with a keratotic scale, or as plaques covered with a multilayered, adherent horny yellowish or white substance, or as diffuse hyperkeratosis looking like chalky dust applied on the skin (Fig. 6) [16]. Notably on the palms and soles, hypertrophic LE causes keratoderma with 1-3 mm thickness that may interfere with the mobility of the fingers.

During the evolution of lesions cicatricial atrophy, at times mutilating, may appear. Characteristic dense lymphocytic infiltrate, especially perivascular, colloid bodies, vacuolar degeneration of the dermoepidermal interface, as well as positive DIF of the basal membrane are relevant to recognize hypertrophic DLE.

Hypertrophic DLE has to be differentiated from hypertrophic lichen planus, ostraceous psoriasis, prurigo nodularis, keratoacanthoma, and epithelial carcinoma [2]. Notably, hypertrophic LE may present also histologically as pseudocarcinomatous hyperplasia that can cause confusion with squamous cell carcinoma.

\section{Lupus Comedonicus}

Lupus comedonicus is an uncommon presentation of DLE: only 13 cases have been reported in the literature, especially in women, in the third decade of life. Comedonal CCLE can be mistaken for benign dermatological conditions, especially acne vulgaris, Favre-Racouchot disease, nevus comedonicus, syringoma, tricoepithelio$\mathrm{ma}$, and agminate lichen follicularis with cysts and comedones, but CCLE bears a highly destructive potential, with mutilations and atrophic scarring occurring in un-

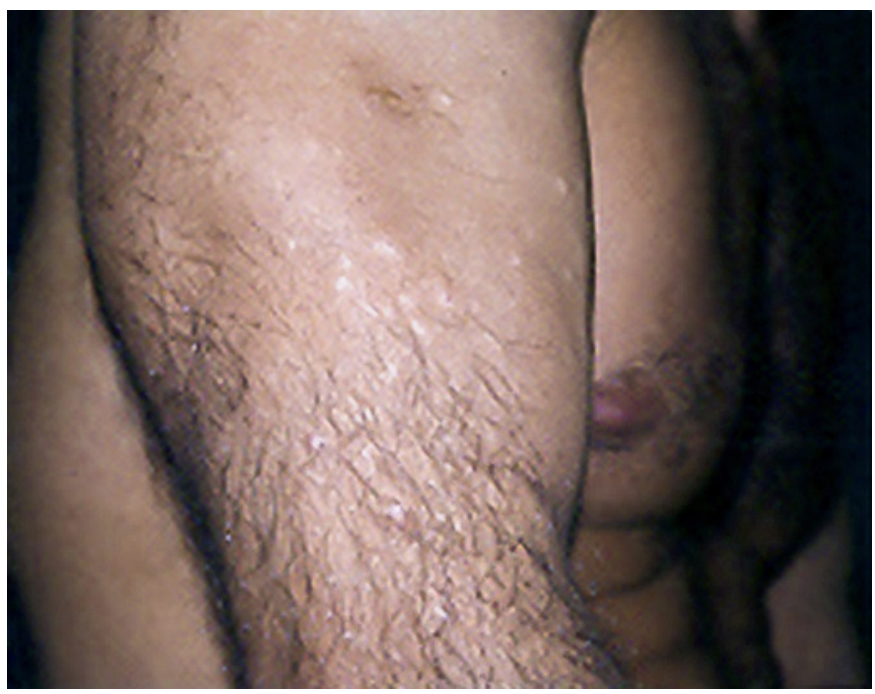

Fig. 8. Papulonodular mucinosis: asymptomatic skin-colored papules.

treated disease. Furthermore, 31\% of comedonal CCLE patients have concomitant SLE. Hystopathology shows acanthosis, vacuolar degeneration of the basal cell layer, periadnexial mononuclear infiltrate, follicular plugging, and comedones [17].

\section{Lupus Mastitis}

Lupus erythematosus profundus is a well-described variant of CCLE also known as lupus erythematosus panniculitis. However, breast involvement of lupus profundus is very rare, less than 30 cases having been reported in the literature. It is defined as lupus mastitis (Fig. 7). It can occur in patients ranging in age from 18 to 70 years and usually occurs in females. The course of the disease tends to be chronic with remission and flares [18].

Lobular panniculitis is characteristic of lupus profundus with dense lymphocytic, plasmacellular, and histiocytic patchy infiltrate, accompanied by interface dermatitis when associated with overlying DLE, mucin deposition, necrotic adipocytes, sclerotic healing, and hyalinization of connective tissue separating fat lobules. DIF shows deposits of IgG, IgM, and C3 in the basal membrane of the overlying skin $[16,18]$.

\section{Other Forms of Atypical CLE}

These forms of CLE may present a rapid or more gradual cutaneous progression and comprehend a spectrum of clinical and histological manifestations from ACLE to 
SCLE and DLE and a more or less prominent systemic involvement not allowing to properly classify them into one of the previous categories (Table 1).

\section{Papulonodular Mucinosis in LE}

Clinically, papulonodular mucinosis presents as asymptomatic skin-colored papules and nodules that typically involve the trunk and upper limbs (Fig. 8), but other areas may also be affected. In about $80 \%$ of cases it is associated with SLE. About half of the patients with papulonodular mucinosis have associated renal disease and more than half have arthritis.

Histologically only deposits of mucin are visible in the superficial and mid-dermis associated with a slight to moderately dense perivascular lymphocytic infiltrate and normal epidermis. Typical interface changes are not present. Typical linear or granular deposits of IgG, IgM, and $\mathrm{C} 3$ at the dermal-epidermal junction are usually present.

Some authors describe a variant of mucinosis in LE named reticular-type erythematous mucinosis: the clinical presentation is characteristic with net-like arrangement of erythematous papules and plaques on the chest and back. Similar to lupus erythematosus tumidus, reticular-type erythematous mucinosis presents remarkable photosensitivity and histologically a dense lymphocytic infiltrate and mucin deposits [2].

\section{Linear LE}

Linear LE (LE linearis) is a very rare variant of CLE presenting along the Blaschko lines, which are pathways of epidermal cell migration during fetal development and reflect the existence of genetic mosaicism. Liner LE is described mostly in the pediatric age and in young adults, with facial predilection and less frequently involving the trunk and limbs. It comprehends a spectrum of clinical and histological manifestations from ACLE to SCLE, DLE, lupus erythematodes tumidus, bullous LE, and lupus erythematosus panniculitis. However, the histopathological findings of linear LE lesions are mostly consistent with those of DLE, and traumatic skin injury and UV light were observed to induce linear lupus lesions (Koebner phenomenon or isomorphic response) [5]. Differential diagnoses that must be considered are linear morphea, linear lichen planus, linear verrucous epidermal nevus, and linear psoriasis [19].

\section{Monogenic LE}

Monogenic LE (MLE) is a form of LE occurring due to single gene mutations. It is a rare variant of lupus that has provided relevant insights into the pathogenesis of SLE [20]. Indeed, a very high number of genes are implicated

Atypical Cutaneous Lupus Erythematosus in MLE, defining MLE forms related to inherited complement deficiencies, forms related to altered nucleic acid repair, degradation, and sensing, as well as forms related to dysregulation of the type I interferon pathway and to B cell development checkpoints [21].

MLE typically presents with an early onset, within the first 5 years of life, with severe clinical manifestations especially affecting the central nervous system, the kidneys, joints, and skin [20]. Among MLE, familial chilblain lupus is characterized by the presence of painful bluish-red papular or nodular lesions of the skin in acral locations (fingers, toes, nose, cheeks, and ears) that tend to ulcerate.

Familial chilblain lupus usually begins in early childhood and is precipitated by cold and wet exposure. Apart from arthralgias, there is no evidence of systemic disease. Histology shows a deep inflammatory infiltrate with perivascular distribution and granular deposits of immunoglobulins and complement along the basement membrane [22].

\section{Toxic Epidermal Necrolysis-Like LE and Rowell Syndrome}

Particularly severe and even life-threatening, but fortunately rare forms of CLE are toxic epidermal necrolysis (TEN)-like LE and erythema exsudativum multiforme (EEM)-like LE also known as Rowell syndrome. Classically TEN and EEM are associated with drug reactions and, especially EEM, also with herpesvirus and Mycoplasma pneumoniae infections. However, both have been reported in association with SLE, ACLE, and SCLE, in the absence of triggering drugs or infections.

TEN-like LE consists in acute necrosis of the epidermis, causing widespread detachment of the skin (positive Nikolsky sign) and of the mucous membranes. Presumably, in these cases TEN was triggered by disease flares, e.g., following intensive UV exposure, sometimes presenting an unusual subacute course over several weeks [6].

EEM may be observed in patients with all subtypes of CLE (ACLE, SCLE, and DLE) and SLE. It presents with recurrent cutaneous EEM-like lesions and is characterized by speckled ANA as well as possible anti-Ro or antiLa antibodies and rheumatoid factor [23, 24]. It has been suggested that EEM-like lesions and speckled ANA pattern should be present as major criteria, in addition to one of the minor criteria (chilblain-type lesions, anti-Ro or anti-La antibodies, or positive rheumatoid factor) to make a diagnosis of Rowell's syndrome [25]. Classical EEM triggers, such as HSV or M. pneumoniae infection and ingestion of high-risk drugs, must be ruled out [2325]. 


\section{Conclusion}

It becomes clear how important the role of the clinician is in diagnosing LE and how relevant it is for the dermatologist to know and be able to suspect and possibly diagnose even the rarest forms of CLE, with the relevant support of immunofluorescence usually showing the typical linear or granular deposits of IgG, IgM, and C3 at the dermal-epidermal junction (lupus band) of involved and uninvolved skin in SLE and of involved skin only in CLE without systemic involvement [26].

\section{Key Message}

Atypical cutaneous manifestations are rare but characteristic, enabling an educated practitioner to suspect LE.

\section{Statement of Ethics}

We obtained the patients' written informed consent for publication of their photos.

\section{Conflict of Interest Statement}

The authors have no conflicts of interest to declare.

\section{Funding Sources}

None.

\section{Author Contributions}

A. Herzum: conceptualization, data curation, formal analysis, investigation, methodology, project administration, resources, software, supervision, validation, visualization, writing - original draft, writing - review and editing. G. Gasparini: conceptualization, data curation, formal analysis, investigation, methodology, project administration, resources, software, supervision, validation, visualization, writing - original draft, writing - review and editing. M. Burlando: conceptualization, data curation, project administration, supervision, validation, visualization, writing - review and editing. E. Cozzani: conceptualization, data curation, project administration, supervision, validation, visualization, writing - review and editing. A. Parodi: conceptualization, data curation, project administration, supervision, validation, visualization, writing - review and editing.

\section{References}

1 Wenzel J. Cutaneous lupus erythematosus: new insights into pathogenesis and therapeutic strategies. Nat Rev Rheumatol. 2019;15: 519-32.

2 Obermoser G, Zelger B, Zelger B. Lupus erythematodes - ein klinisch-pathologisch heterogenes Krankheitsbild. Pathologe. 2020; 41(4):334-43.

3 Gilliam JN, Sontheimer RD. Distinctive cutaneous subsets in the spectrum of lupus erythematosus. J Am Acad Dermatol. 1981;4:471-5.

4 Werth VP. Clinical manifestations of cutaneous lupus erythematosus. Autoimmun Rev. 2005;4(5):296-302.

5 Obermoser G, Sontheimer RD, Zelger B. Overview of common, rare and atypical manifestations of cutaneous lupus erythematosus and histopathological correlates. Lupus. 2010; 19:1050-70.

6 Ting W, Stone MS, Racila D, Scofield RH, Sontheimer RD. Toxic epidermal necrolysislike acute cutaneous lupus erythematosus and the spectrum of the acute syndrome of apoptotic panepidermolysis (ASAP): a case report, concept review and proposal for new classification of lupus erythematosus vesiculobullous skin lesions. Lupus. 2004;13:94150.

7 Da Silva Sousa AC, Campos M, Oliveira A, Menezes N, Tente D, Baptista A. Bullous lupus erythematosus with an erythema gyratum repens-like pattern. Dermatol Online J. 2019; 25:13030/qt0nt6h49v.
8 Kalavala M, Shah V, Blackford S. Subacute cutaneous lupus erythematosus presenting as erythroderma. Clin Exp Dermatol. 2007;32:388-90.

9 Kreft B, Marsch WC. Lupus erythematosus gyratus repens. Eur J Dermatol. 2007;17:7982.

10 Chander R, Yadav P, Singh A, Nangia A. Systemic lupus erythematosus presenting as erythema annulare centrifugum. Lupus. 2014;23: 1197-200.

11 Parodi A, Cozzani E. Cutaneous manifestations of lupus erythematosus. G Ital Dermatol Venereol. 2014;149:549-54.

12 Rothfield N, Sontheimer R, Bernstein M. Lupus erythematosus: systemic and cutaneous manifestations. Clin Dermatol. 2006;24:348-62.

13 Chong BF, Song J, Olsen NJ. Determining risk factors for developing systemic lupus erythematosus in patients with discoid lupus erythematosus. Br J Dermatol. 2012;166:29-35.

14 Devergie MGA. Traité pratique des maladies de la peau. Paris: Librairie de Victor Masson; 1854.

15 Bechet PE. Lupus erythematosus hypertrophicus et profundus; a further attempt to elucidate its status. Arch Derm Syphilol. 1950; 61(3):495-8

16 Mascaro JM, Herrero C, Hausmann G. Uncommon cutaneous manifestations of lupus erythematosus. Lupus. 1997;6:122-31.

17 Cozzani E, Herzum A, Burlando M, Parodi A. Comedonal variant of chronic cutaneous lupus erythematosus causing mutilation of the earlobe. JAAD Case Rep. 2020;6:843-4.
18 Voizard B, Lalonde L, Sanchez LM, RichardChesnay J, David J, Labelle M, et al. Lupus mastitis as a first manifestation of systemic disease: about two cases with a review of the literature. Eur J Radiol. 2017;92:124-31.

19 Saha A, Seth J, Pradhan S, Dattaroy S. Disseminated discoid lupus erythematosus with a linear lesion on the forearm and hand: a rare presentation and review of literature. Indian J Dermatol Venereol Leprol. 2017;83:586-9.

20 Alperin JM, Ortiz-Fernández L, Sawalha AH Monogenic lupus: a developing paradigm of disease. Front Immunol. 2018;9:2496.

21 Costa-Reis P, Sullivan KE. Monogenic lupus: it's all new! Curr Opin Immunol. 2017;49:87-95.

22 Lee-Kirsch MA, Gong M, Schulz H, Rüschendorf F, Stein A, Pfeiffer C, et al . Familial chilblain lupus, a monogenic form of cutaneous lupus erythematosus, maps to chromosome 3p. Am J Hum Genet. 2006;79:731-7.

23 Shteyngarts AR, Warner MR, Camisa C. Lupus erythematosus associated with erythema multiforme: does Rowell's syndrome exist? J Am Acad Dermatol. 1999;40:773-7.

24 Rowell NR, Beck JS, Anderson JR. Lupus erythematosus and erythema multiforme-like lesions. A syndrome with characteristic immunological abnormalities. Arch Dermatol. 1963;88:176-80.

25 Zeitouni NC, Funaro D, Cloutier RA, Gagne E, Claveau J. Redefining Rowell's syndrome. Br J Dermatol. 2000;142:343-6.

26 Monroe EW. Lupus band test. Arch Dermatol. 1977;113(6):830-4. 